\title{
CONTANDO HISTORIAS PARA CUIDAR Y TRANSFORMAR
}

\author{
TELLING STORIES TO CARE AND TRANSFORM
}

CONTANDO HISTÓRIAS PARA CUIDAR E TRANSFORMAR

\author{
Mirliana Ramírez-Pereira \\ Departamento de Enfermería \\ Universidad de Chile \\ mirliana@uchile.cl \\ ORCID:0000-0003-1439-4162
}

Esmerita Opazo Morales
Departamento de Enfermería
Universidad de Chile
ORCID: 0000-0003-3061-1390

DOI: https://doi.org/10.22235/ech.v7i2.1660

Recibido: 20/04/2018

Aceptado: 30/08/2018

\begin{abstract}
Resumen: La postmodernidad ha traído consigo individualismo, globalización y fragmentación del tejido social. Enfermería en su visión holística debe mantener el cuidado integral de la persona familia y comunidad, sin perder la conexión consigo misma y con el entorno social. El objetivo de este ensayo fue reflexionar acerca de las emociones, sentimientos y pensamientos que emergen en la enfermera-investigadora, cuando se explora el cuidado y las representacionales sociales de las personas con enfermedad renal. Como metodología se usó la autoetnografía interpretativa, como una propuesta metodológica que rompe con la dinámica de enfermería tradicional. Como conclusión hemos podido observar el vínculo existente entre la experiencia de la enfermedad, de la investigación cualitativa y del cuidado desde la perspectiva del paciente y la enfermera, validando la metodología de la autoetnografía interpretativa como una forma de mejorar la comprensión de los fenómenos asociados al cuidado de enfermería.
\end{abstract}

Palabras Clave: Enfermería, Insuficiencia renal, Investigación Cualitativa, Enfermedad crónica.

\begin{abstract}
Postmodernity has come with individualism, globalization, and destruction of social networks. The nurse, with her holistic care, must kept the health care of the person, the family, and the community without losing the connection with herself. The aim of this piece was to reflect on the emotions, feelings and ideas that arise from the nurse-researcher to the care and the study of social representations of end stage renal disease. The method was interpretative autoethnography, as a methodological proposal that breaks with the traditional dynamic of the nursing. Our conclusion was that there is a connection between
\end{abstract}


the experience of the disease, nursing care and the qualitative research of patients and nurses, confirming interpretative autoethnography as a way of understanding the phenomena associated with nursing care.

Keywords: Nursing, Renal Insufficiency, Qualitative research, Chronic disease.

Resumo: O pós-modernismo trouxe consigo o individualismo, a globalização e a fragmentação do tecido social. A enfermagem em sua visão holística deve manter o cuidado integral da pessoa família e comunidade, sem perder a conexão consigo mesma e com o meio social. O objetivo deste ensaio foi refletir sobre as emoções, sentimentos e pensamentos que emergem no enfermeiro-pesquisador, quando se exploram os cuidados e representações sociais de pessoas com doença renal. Como metodologia, utilizou-se a autoetnografia interpretativa como proposta metodológica que rompe com a dinâmica tradicional da enfermagem. Em conclusão, vimos a ligação entre a experiência da doença, a pesquisa qualitativa e cuidado do ponto de vista do paciente e a enfermeira, validando a metodologia de autoethnography interpretativa como uma maneira de melhorar a compreensão dos fenómenos associados cuidados de enfermagem.

Palavra chave: Enfermagem, Pesquisa qualitativa, Insuficiencia Renal Cronica, Doença Cronica.

\section{INTRODUCCIÓN}

La mayor parte de las historias de las enfermeras se pierden en el cuidado de los enfermos y de los postergados. No porque no tengan valor, sino porque que no están escritas, solo quedan en el recuerdo de aquellos que tuvieron el privilegio de ser atendidos con amor y sabiduría. No se escriben y ciertamente no están presentes en los libros de Historia Universal, olvidando de esta forma la pequeña historia, la del día a día, aquella que es la historia de todas y que se construye con los acontecimientos de la vida. Cuando las enfermeras rescatamos y narramos las historias de nuestros pacientes, que inevitablemente se vinculan a la propia, desafiamos los estándares de la investigación tradicional, de aquella investigación cuya premisa parte con que la realidad es objetiva, que puede ser aprehendida y medible.

¿Pero qué ocurre cuando la historia va más allá, cuando no solo rescata la narrativa del otro, sino que también la propia? ¿Cuándo el investigador no niega, sino que, por el contrario, reivindica la subjetividad y su propia experiencia, enfatizando en los aspectos sociales que acompañan a la enfermedad, la salud y el cuidado? Es en esa instancia de reconocerse en el otro, en el comprender que nos une a enfermeras y pacientes la misma dignidad y humanidad, que todos compartimos vivencias, historia y cultura, es cuando podemos vislumbrar la enfermería como herramienta de transformación social.

En este camino de transformación social surge la autoetnografía como un método y una posibilidad de encontrarse con el yo-enfermero, para entregar el cuidado humanizado que los pacientes requieren de los profesionales de la salud, especialmente de las enfermeras, las cuales deben poseer habilidades para relacionarse con ellos, para darles la bienvenida 
con humanidad, para entender sus miedos, identificándose con su dolor, ofreciendo seguridad, enfermeras que sean capaces de observar y que permitan ser observadas (1).

En un sentido más amplio, si comprendemos la investigación cualitativa como un cristal de múltiples caras, con un discurso energizante, rebelde, donde se mezcla el arte y la ciencia y en el cual se combinan ambas con una infinita variedad de formas, sustancias y transmutaciones, podremos comprender que la realidad es mucho más de lo que vemos con una ilusión de capturar la realidad objetiva. En este contexto multidimensional, multifásico y de múltiples aproximaciones a la realidad, la ciencia de enfermería se posiciona en una interfaz integrativa desde las miradas humanistas, sociales, políticas y espirituales, como una alternativa de holismo y transversalidad (2).

Lyotard plantea que, en la actualidad, el mundo occidental se encuentra en la condición de post modernidad, la cual está dada por un descontento frente a los sistemas, una incredulidad con respecto a los metarelatos de tipo unificador sobre los cuales se sustentó la modernidad (3). El post modernismo está establecido por la globalización, marcado por las comunicaciones y uso de nuevas tecnologías, con un acento en la homogeneización social y multicultural, con lo que se ha desaparecido la concepción de ser humano, familia y comunidad tradicional de la época premoderna, comprendidas como holísticas, centradas en el ser y obedientes a los ciclos naturales (4). Estos cambios en que el neoliberalismo se ha impuesto involucran a todos los países en occidente. Uno de los más afectado ha sido Chile, por su condición de laboratorio de un modelo neoliberal, que consolidó transformaciones sociales, económicas y políticas que se mantienen hasta nuestros días, con las cual se ha profundizado la desigualdad social con un índice de Gini de 0,5 , uno de los más altos de la Organization for Economic Cooperation and Development $(5,6)$.

La enfermedad renal crónica es considerada un problema mundial que sobrecarga los sistemas de salud y se traduce en millones de muertes y años de vida perdidos. Los cambios epidemiológicos a nivel mundial también se reflejan en la salud de los chilenos, con un aumento importante en la prevalencia e incidencia de las enfermedades crónicas no transmisibles (7). Dentro de éstas, la enfermedad renal se ha incrementado en forma considerable: al igual que las patologías que son factores de riesgo para ella, como son las diabetes e hipertensión arterial crónica. La enfermedad renal en etapa $\mathrm{V}$ afecta a 19.071 pacientes en diálisis, con un 45,5\% de mujeres y un $51 \%$ del total con más de 60 años. Un $88 \%$ de ellos se dializan en centros de diálisis privados y un $12 \%$ en clínicas y hospitales públicos (8).

Es importante recordar que la enfermedad renal crónica no sólo afecta a un determinado órgano del cuerpo; sino que elige al cuerpo mismo como medio de expresión con el mundo, sin poder separar las partes sanas del mismo (9). Esta enfermedad se traduce en un costo personal, familiar y social importante con niveles altos de ansiedad, depresión, ideas suicidas y desprecio hacia sí mismos (10). Esto se ve agravado por la baja tasa de ocupación de los usuarios en terapias de sustitución renal; según Julián-Mauro sólo el 33,3\% desarrollan algún tipo de actividad remunerada (11).

Los grandes procesos mencionados y las características que estos imprimen o retroalimentan en las personas, familias y comunidades, generan inmensos cambios en distintas dimensiones de la vida cotidiana influyendo y complejizando todas las ciencias. Sin embargo, ciertos cambios en la comprensión del ser humano se venían dibujando desde 
hace siglos. Según Le Breton la ruptura en la concepción integral del ser humano se produce con los primeros anatomistas, quienes, al diseccionar el cuerpo, transformando a la persona en un objeto (12).

Este artículo nace de las notas de campo, vivencias y recuerdos de las entrevistas a personas con enfermedad renal crónica, en el marco de la tesis doctoral "Humanizar el cuidado: comprendiendo las representaciones sociales de la enfermedad renal en personas en diálisis", rescatando de esta forma la intersubjetividad de la relación enfermera-paciente, como así también la voz de quienes sufren la enfermedad.

\section{METODOLOGÍA}

El propósito de esta investigación fue reflexionar acerca de las emociones, sentimientos y pensamientos que emergen en la enfermera-investigadora, cuando se explora el cuidado y las representaciones sociales respecto a su padecimiento de las personas con enfermedad renal, comprendiendo las representaciones sociales como un "sistemas de opiniones, de conocimientos y de creencias propias de una cultura, una categoría o un grupo social y relativas a objetos del contexto social" (13).

La metodología utilizada fue cualitativa, ya que permite comprender los significados, otorgando una importancia contextual, sin perder de vista los aspectos particulares de la situación a estudiar. Esto se relaciona fundamentalmente con la idea de búsqueda de sentido, con el fin de lograr la comprensión de los fenómenos, resultando incluso más importante que la explicación de un fenómeno particular (14).

El paradigma utilizado fue el constructivista. Según este paradigma las personas significan e interpretan los fenómenos según su historia y su cultura. El paradigma constructivista reconocer que el mundo real se construye en base a interaccionismo social y recursos representacionales (15).

La perspectiva utilizada fue la autoetnografía interpretativa, la que se comprende como el uso de la experiencia y escritura personal para comentar las vivencias y prácticas culturales, denunciar la vulnerabilidad y crear relaciones recíprocas con quienes leen con el fin de lograr una identificación con la historia (16-17). Según esto, las personas crean su vida por medio de historias de quienes son ellos y los otros, interpretando su vida según estas historias (18).

Esta investigación se basa en los principios de ética comunicativa de Habermas, según los cuales, interlocutores son validados como iguales, en que ambos tienen voz e historias que contar (19). La participante firmo un consentimiento informado, en el que autorizaba la entrevista, la grabación y transcripción, además de su uso en publicaciones académicas, resguardando el anonimato. La entrevista se realizó en el hogar de la participante, con una duración aproximada de 90 minutos. 


\section{DESARROLLO}

Caminante no hay camino, se hace camino al andar

(Proverbios y cantares (XXIX), Antonio Machado)

Soy una enfermera especialista en enfermería nefrourológica. He trabajado durante largo tiempo en el cuidado de personas con enfermedad renal, actividad que he realizado en conjunto con la docencia de pre y post grado. Durante todos estos años he tenido siempre presente que los seres humanos somos integrales. En este camino me he contactado con el sufrimiento de mis pacientes, con lo duro que resulta para ellos padecer esa enfermedad, de lo difícil que es insertarse en el mundo laboral y social y de los problemas que surgen al interior de las familias.

Luego de terminar mi Máster en Psicología Comunitaria, decidí realizar el Doctorado en Enfermería e investigar el cuidado y las representaciones sociales de las personas con enfermedad renal, para así poder visibilizar desde el sentido común de los pacientes como comprenden la enfermedad renal, como les afecta emocionalmente y cuál es su campo de representación.

Muchas veces en el cuidado de la persona enferma renal se prioriza lo biológico, restando importancia a lo psicológico, social y espiritual, llegando a ver al hombre como una máquina, olvidándonos de la integralidad y del sufrimiento de él y su familia.

$* * * *$

Después de revisar innumerables documentos, artículos, capítulos de libros, analizar y reflexionar sobre nuevas ideas, pasar horas encerrada en mi oficina, intentando vincular el cuidado humanizado y las representaciones sociales, reflexionando y pensando para así poder plasmarlas en un proyecto de investigación, por fin llega el esperado trabajo de campo.

Una agradable sensación de alegría me invade, es la oportunidad de salir de las largas horas de encierro y aislamiento para poder acercarme a los pacientes, conocer de forma intencionada su realidad, su vida. En un Chile donde la desigualdad social es parte de nuestra vida, esta instancia se convierte en una posibilidad de salir de mi zona de confort de enfermera, de esposa y de madre y conocer más profundamente a mis paciente, explorar sus inquietudes respecto a su enfermedad, todo ello me lleva a imaginar un escenario inquietante y motivador, durante largo tiempo preparando el proyecto, imagino que el trabajo de campo será muy enriquecedor para mí y espero que así lo sea también para las personas que sufren esta enfermedad. La investigación se realizará en Coquimbo, pequeña ciudad donde vivo, que se ubica a 471 kilómetros al norte de Santiago, la capital de Chile. Busco en un mapa la dirección de mi primera entrevistada, la Sra. María (nombre ficticio). Debo ingresar a un sector que no conocía de la ciudad, casas muy humildes de madera, pero limpias y ordenadas. Observo algunos jóvenes conversando en la calle, con música a alto volumen, son las $15 \mathrm{hrs}$, pienso en la falta de oportunidades que en mi país parte desde la cuna, en el sentido de que los niños crecen marginados en guetos de pobreza, con educación deficiente, sin áreas verdes donde jugar ni espacios públicos seguros.

Toco a la puerta, y me recibe una mujer de unos 70 años, dulce y cariñosa, me presento y me da un beso en la mejilla. Su casa es pequeña, con adornos y cuadros por todas partes, 
fotografías familiares y regalos. Me explica que vive con un nieto que trabaja todo el día y que tiene familiares que viven cerca. Le comento el objetivo de la entrevista, acepta sin reparos y firma el consentimiento informado con el cual me autoriza a realizarla.

Me cuenta que a pesar de su enfermedad ella es una persona feliz, que se siente amada y cuidada por todos, en especial por las enfermeras de su clínica de diálisis, me dice que están siempre pendientes de ella: si le baja la presión arterial o sufre de calambres, que les preguntan por su familia y también le cuentan aspectos de su vida fuera de la enfermería. Insiste que todo esto es con "mucho respeto". Mientras habla, la observo, su rostro curtido por el sol del norte de Chile, un poco pálida, con arrugas profundas que cubren su cara, pero con una sonrisa maravillosa que inunda la habitación. Desde mi mirada considero que es una experiencia terrible la diálisis, acudir tres veces a la semana, perder 4 horas cada vez, para conectarse a la máquina que la mantiene con vida. Observo el brazo de su fistula arterio-venosa, algo edematoso, sus manos de mujer trabajadora, su ropa humilde, que da cuenta de su origen social. Escucho sus palabras siempre iluminada con una sonrisa. Le digo, cuénteme su historia, recuerdo las palabras de uno de mis profesores que enfatizaba que la mejor entrevista es la conversación de amigos, dejo de lado mi uniforme blanco y con esto el traje de experto y la escucho:

"Llegue sólo a 4to año de educación primaria, quede huérfana a los 8 años, me crio mi papá, es triste crecer sin madre, sin nadie que te oriente y cuide. En esa búsqueda de amor, quedé embarazada a los 14 años, de mi esposo. Fui muy maltratada por él, me golpeaba todos los días. Cuando enviudé, a los 46 años, decidí no tener nunca más una pareja, crie sola a mis cinco hijos".

Las palabras de mi entrevistada brotan sin dificultad, parece que hubiera esperado mucho tiempo para contar su historia. No puedo evitar sentir compasión por ella y por tantas otras mujeres que cargan con el estigma de la soledad y la pobreza y se refugian en las parejas para sentirse amadas, tolerando violencia física y psicológica.

Continua con sus palabras, a veces como si yo no estuviera presente. Me cuenta que es católica y que hasta antes de enfermar iba todos los domingos a misa. Actualmente ya no puede, refiere no tener ánimos ni fuerzas para ir, Aunque enfatiza, que sigue siendo muy creyente:

"Tengo una imagen de Jesús junto a mi cama, me persigno antes de salir a cualquier lugar, le pido fuerzas, solo vivo por él. Las enfermedades son cosas que manda Dios, son pruebas que manda nuestro señor para creer que el existe, yo ya estoy resignada, es lo que me toco y si no me ha llevado es que todavía sirvo acá”.

Como enfermera he escuchado muchas veces esta explicación, esta forma de comprender la enfermedad como algo enviado por Dios, a veces como prueba, otras como castigo. Me observo y recuerdo que muchas veces durante mi vida he explicado los acontecimientos bajo los mismos preceptos, como algo necesario para fortalecerme o como pruebas de la vida para poder crecer. Evoco los fríos inviernos en Santiago, cuando mi hijo pequeño sufrió bronconeumonía, miraba su rostro con una mascarilla de oxígeno y pensaba: si Dios me envió este hijo no va a permitir que muera, quizás sea una prueba de Él, para ver si tengo fe, si sigo creyendo. Pienso que de una $u$ otra manera esta forma de entender las enfermedades y acontecimientos otorga paz y confort dentro de todo el sufrimiento que 
significa el estar enfermo. Latinoamérica es profundamente religiosa, el cristianismo es parte del día a día y guía las vidas de los pueblos, es parte de nuestra cultura aceptar el sufrimiento y el sacrificio como parte de la vida.

María dice: "Mi señorita, mi vida ha estado llena de sufrimientos; cuando tenía 25 años, me dio una forma muy grave de difteria, que me dejo postrada por 8 meses en cama, tuve que volver a aprender a caminar y moverme. Me trataron en Santiago, aquí no había los avances para curarme. Luego trabaje por turnos en la empresa pesquera que había en Coquimbo, en invierno con mucho frio debía enlatar los mariscos y pescados, quizás por eso dejaron de funcionar mis riñones". Continua con su historia: "yo no me he portado mal, no he sido mala, nunca fui una mujer que saliera con las amigas a divertirme en las noches".

La escucho y vuelvo a pensar en los estereotipos y prejuicios que rodean el ser mujer "las malas mujeres salen de noche, con amigas, las buenas se quedan en casa". Reflexiono en esta última frase y aflora el recuerdo de la investigación que estamos desarrollando en algunos países latinoamericanos acerca de las representaciones sociales de género en niñas y niños en edad escolar, los discursos de niñas de 10 años son los mismos de las mujeres de 70 años en Chile, "las niñas buenas se quedan en casa y ayudan a la mama en los quehaceres del hogar" (20).

María continua con su relato: "hace 1 año y 5 meses que estoy en diálisis", me cuenta que llego al servicio de urgencia, “con problemas para respirar y el potasio muy alto”. Me cuenta que sabe que no tiene cura y que por su edad es difícil se pueda trasplantar. María está informada, tiene razón al aseverar esto, los chilenos somos muy solidarios cuando se trata de catástrofes y todos somos donantes por una ley que así lo dicta, sin embargo, en la práctica las personas esperan un milagro que salve a su ser querido y se pierden horas sumamente valiosas que imposibilitan el trasplante. Lo vi muchas veces en las Unidades de Cuidados Intensivos, cuando el equipo de procuramiento dedicaba tiempo y amor a su labor sin resultados, observaba mientras hemofiltraba a los pacientes, intentando dar algo de consuelo a la familia, sin interferir en el trabajo de la enfermera de trasplante y sin juzgar a la familia, para no entorpecer mi cuidado.

Me cuenta que es feliz cuando debe ir a la clínica a dializarse, se siente acompañada por los otros pacientes y enfermeras, para ella son su familia y refugio. La escuchan, acogen y acompañan, comenta que trata de no ser una molestia para nadie, que solo cuando se siente muy mal avisa al personal de salud, que intenta no crear conflictos y llevar una vida tranquila.

Exclama: "Señorita le agradezco tanto el que haya venido a visitarme y que escuche mi historia, a veces me siento tan sola, solo la televisión me acompaña”. Me ofrece tomar una taza de té con ella. Siento un poco de vergüenza y culpa, los adultos mayores en Chile reciben una pensión muy baja. Lo acepto y huelo el té recién hecho, acerco la taza a mis labios y mientras bebo un sorbo, la observo, con su maravillosa sonrisa, a pesar de su vida tan dura, veo su mezcla de resignación y deseos de seguir viviendo, recuerdo sus palabras: "para qué voy a tener rabia con la vida y es lo que el señor quiso para mí". Le agradezco su tiempo, su alegría y la taza de té, debo dejarla para atender a dos pacientes nefrópatas en la unidad de cuidados intensivos. Me alejo de su casa, mientras ella me dice adiós con su mano, la veo feliz y tranquila. Mientras conduzco hasta el hospital, las sensaciones de 
frustración y tristeza por no poder hacer más por ella se desvanecen, me relajo, unas lágrimas caen por mi rostro, efímeras, pero cargadas de fuerza, y como dice nuestra gran Violeta Parra; le doy "gracias a la vida que me ha dado tanto".

\section{REFLEXIONES FINALES}

Los seres humanos somos sociales y dependientes de otras personas. Esta dependencia requiere una respuesta humana en forma de cuidado, tomando conciencia de que el estar con el otro, provoca reacciones en ambos, según esto, la situación de cuidado es contextual, emocionalmente cargada y particularista en su naturaleza, comprendiendo el cuidado como un ser material y presente en una relación con nuestros sentidos y nuestros cuerpos (21).

Por otro lado, Waldow refiere que las enfermeras no tenemos consciencia del poder del cuidado ni del poder que las actividades de Enfermería con base en la acción de cuidar. Esto se debe, en parte, a una infravaloración del cuidado por parte de la propia enfermera. El conocimiento está vinculado al poder, al hacer sabiendo y hacer mejor y desde esa perspectiva, el cuidado puede ser político y emancipador (22).

Pires plantea un triedro emancipatorio del cuidar: conocer para cuidar mejor, cuidar para confrontar y cuidar para emancipar, queda claro la articulación entre conocimiento, poder y autonomía (23). El poder transformador de la enfermera esta dado por la posibilidad de cuidar rescatando la subjetividad, empoderando y emancipando a la persona, entregando un espacio a la voz de los silenciados e invisibles, como han sido los "pacientes" desde la biomedicina.

$* * * * * * *$

La enfermedad es una marca oscura en la vida de María, es una cadena que la mantiene en la sombra, después de una vida determinada por el abandono y el maltrato, sufrir de enfermedad renal crónica y dializarse es solo una etapa más, llegando a ser una instancia de aminorar el impacto de la soledad. María está lejos de la democratización del conocimiento que entrega la internet, pertenece a la generación de mujeres que crecieron bajo el yugo de la pobreza, de un esposo maltratador y que ahora se enfrenta a una enfermedad que trae múltiples consecuencias en todos los aspectos de la vida. A pesar del ensombrecido panorama surge la resiliencia como esa capacidad propia de las mujeres de salir adelante, criar a sus hijos y ver los aspectos positivos de la vida.

Cuando escribo reviso mis procesos frente a lo que voy viviendo, como mujer, madre, enfermera, me muestro, me expongo, quizás me debilito a ojos de los demás. Sin embargo, para mi es una instancia en que me humanizo y me sano y puedo ser una enfermera más empática. También, tengo una voz propia, silenciada por cientos de años de sumisión. Una voz que comprende los procesos ciudadanos, comunitarios, sociales e históricos de mi país que son parte de mi propia historia. Puedo hacer narrativa situada, desafiando los cánones tradicionales de hacer academia y de hacer enfermería que años se ha construido desde una mirada biomédica y positivista. En este relato hay un estímulo para crear una nueva enfermería que está naciendo sin permiso, junto con la necesidad imperiosa de transformación social que estalla en cada rincón de Chile. En los tiempos violentos que vivimos, de injusticia social, de silencios forzados para evitar conflictos, de no reconocer al 
otro como un igual, Enfermería está despertando y perdiendo el miedo a hablar, con una voz humanista, social y comprometida con los procesos históricos de los pueblos.

\section{REFERENCIAS BIBLOGRÁFICAS}

1. Corban,I N. Humanização do cuidado de enfermagem: o que é isso? Rev Bras Enferm. 2009;62(3): 349-54

2. Ellingson L. Analysis and Representation Across the Continuum. En: En: Denzin N, The SAGE Handbook of Qualitative Research. London: SAGE, 2011. P. 595-767.

3. Lyotard JF. La condición postmoderna: informe sobre el saber. Madrid: Cátedra; 1982.

4. Rozas G, Pardo M, Yáñez L. Efectos de la modernidad y la postmodernidad en la comunidad indígena Licarantay o Atacameña de San Pedro de Atacama. Psicología para América Latina [internet].2008 [Citado 25 Jul 2018]; 12(1):0-0 Disponible en http://pepsic.bvsalud.org/scielo.php?script $=$ sci_arttext\&pid $=\mathrm{S} 1870$ 350X2008000100004\&lng=pt\&tlng=es.

5. Saavedra E. El Modelo Económico-Político de Chile: Desarrollo Institucional en la Encrucijada. Economía y Política. 2014; 1(1): 117-148.

6. Organisation for Economic Co-operation and Development: OCDE: In It Together Why Less Inequality Benefits All in Chile. [Internet]. Consultado 5 May 2018] Disponible en: http://www.oecd.org/chile/OECD2015-In-It-Together-Highlights-Chile.pdf . Updated 2015.

7. Martin-Cleary C, Ortiz A. CKD hotspots around the world: where, why, and what the lesson are. Clin Kidney J. 2014;7 (6):519-23.

8. Poblete H. XXXV Cuenta de hemodiálisis crónica (HDC) en Chile. [Internet].2015 [citado 17 de julio 2017]. Disponible en: http://www.nefro.cl/site/biblio/registro/21.pdf

9. Rosa KR, Loures MC. Qualidade de vida de idosos em hemodiálise: Enfermagem e o lúdico. Estudos. 2013; 40(4): 419-46.

10. Souza F, Oliveira J. Sintomas depressivos e ansiosos no paciente renal crônico em tratamento conservador. Rev. Psicol. Saúde [Internet]. 2017 citado Abril 252018 ] ; 9(3):17-31. Disponible en: http://pepsic.bvsalud.org/scielo.php?script=sci_arttext\&pid=S2177093X2017000300002\&lng=pt. http://dx.doi.org/10.20435/pssa.v9i3.429.

11. Julián-Mauro J. La situación laboral del paciente con enfermedad renal crónica en función del tratamiento sustitutivo renal. Rev Nefrol. 2012;32(4):439-45

12. Le Bretón, D. (1994). "Lo imaginario del cuerpo en la tecnociencia". REIS.1994; 68, Oct-Dic:197- 210

13. Rateau P, Lo Monaco G. La Teoría de las Representaciones Sociales: Orientaciones conceptuales, campos de aplicaciones y método. CES Psicología. 2013; 6 (1): 22 - 42.

14. Cooper K. Qualitative Research in the Post-Modern Era: Context of Qualitative Research. New York: Springer; 2012 
15. Lincoln I. Paradigmatic controversies, contradictions, and emerging confluences, revisited. En: Denzin N, The SAGE Handbook of Qualitative Research. London: SAGE, 2011 p. $97-107$

16. Custer D. Autoethnography as a Transformative Research Method. The Qualitative Report. 2014; 19(37): 1-1

17. Denzin N. Interpretive Autoethnography. California: SAGE; 2014

18. Clandinin J. Engaging in Narrative Inquiry (Developing Qualitative Inquiry). California: SAGE; 2013

19. Moreno. A. Jürgen Habermas: entre la ética del discurso y la ética de la especie. Tópicos. 2008. 35: 93-112

20. Ramírez-Pereira M. Gender, boys, and girls: an approach from holistic care, Paper presented at: Latin American Studies Association Congress; 2016 May 27-30; New York. 2016. P. 181

21. Håkonsen E. Care for Nurses Only? Medicine and the Perceiving Eye. Health Care Anal. 2011. 19 (1) 15-27

22. Waldow R. Cuidado humano: la vulnerabilidad del ser enfermo y su dimensión de trascendencia. Index Enferm. 2014; 23(4):234-238

23. Pires MRGM. Politicidade do cuidado e processo de trabalho em saúde: conhecer para cuidar melhor, cuidar para confrontar, cuidar para emancipar. Cienc Saude Colet. 2005; 10(4):1025-45. 